\title{
STRESS TENSOR OF FREE MAXWELL FIELD IN FRIEDMANN- ROBERTSON-WALKER UNIVERSE
}

\author{
S.K. Sharma*, P.R. Dhungel** and U. Khanal** \\ *BPKM Planetarium, Observatory and Science Museum Development Board, Ministry of Science \& Technology, \\ Singhadurbar, Kathmandu, Nepal. \\ **Central Department of Physics, Tribhuvan University, Kirtipur, Nepal.
}

\begin{abstract}
The solutions of the Maxwellian field in FRW spacetime, found by using the Newman Penrose formalism, is used to determine the energy-momentum tensor. The tensor is obviously traceless, with the energy density equal to the sum of the radial and the two tangential pressures. But it turns out that the radial and tangential pressures are not equal, giving rise to anisotropy. Such anisotropy can be the origin of the rotation of galaxies. Another result is that the photon energy in a closed universe are quantized in units of one from the lowest value of two upwards. The lowest quantum of two can be interpreted as one unit of spin energy and one of translational energy.
\end{abstract}

Key words: Galactic structure; Maxwellian field; Anisotropic pressure.

\section{INTRODUCTION}

Newman-Penrose (NP) formalism ${ }^{1}$ has been very successful in describing the behaviour of quantum spin fields in curved background spacetime ${ }^{2,3,4,5}$. In an earlier work ${ }^{6}$, this method was used to separate the variables of the free Maxwell and Dirac field equations in the Friedmann-Robertson-Walker (FRW) spacetime. Further ivestigation of the massive Dirac field ${ }^{7}$ revealed the radial number distribution which mimics that required by the flat rotation curbe of galaxies. This suggests that the quantum spin fields must be at the heart of the solution of the galactic structure, its rotation, and possibly mass limit. The formalism consists of choosing an appropriate bases of null tetrad to represent the spacetime, and projecting all the field quantities and derivatives onto them. The metric is taken in the form

$d s^{2}=\frac{a(x)^{2}}{K}\left[d x^{2}-d z^{2}-\sin ^{2} z\left(d \theta^{2}+\sin ^{2} \theta d \phi^{2}\right)\right]$,

where the co-ordinates are redefined in relation to that of Ref. [6] as $a d x=\sqrt{K} d t$ and $z=\sqrt{K} r_{*}$; this will allow us to write down single expressions to represent the different cases of the closed, flat and open universes, $\mathrm{K}=(1,0,-1)$ respectively; these different cases will appear in the appropriate limits of $\mathrm{K}$.

The time and the azimuthal parts are separated out as $e^{i(m \phi-\omega x)}$, leaving the angular solutions as Jacobi polynomials ${ }^{8}$ which are not important here, except for the fact that they are orthogonal and hence normalizable. This leaves the radial equation whose solution ${ }^{6,8}$ turn out to be the appropriately weighted Gegenbauer polynomial C:

$R(z)=A \sin ^{l+1} z C_{\omega-l-1}^{l+1}(\cos z)+B \sin ^{-l} z C_{\omega+l}^{-l}(\cos z)$.
Here, $l$ is the spin-weighted angular momentum, i.e. the total angular momentum including the spin; in particular, for the photon field in consideration, its lowest value is 1 . As $0 \leq z \leq \pi$ in Eq. (1.2), it is obvious that the second solution is singular at these ends, so for the regular solution at $\mathrm{z}=0$, we must set $\mathrm{B}=0$. It is important to remember that for the appropriate time dependence, $\omega$ is actually $\omega / \sqrt{K}$. So the solution for the three cases Eq. (1.2) are $\mathrm{z}=\mathrm{r}_{*}$ for closed,the limit $\mathrm{K} \rightarrow 0$ for the flat in which case the solution goes over to the spherical Bessel function as $r j_{l}(\omega r)$, and in the open case by the replacements $\mathrm{z} \rightarrow \mathrm{iz}$ and $\omega \rightarrow \omega / \mathrm{i}$ or sin and cos by the hyperbolic sinh and cosh; in the last case, these replacements mean writing the Gegenbauer polynomials as the hypergeometric function ${ }_{2} \mathrm{~F}_{1}$. Nonetheless, as ${ }_{2} F_{1}$ is analytic function except at the three singularities, we see that we can think of the three different solutions as analytic continuation throughout the complex z-plane, whence the closed universe lies on the real line, open on the imaginary line and flat near $\mathrm{z}=0$.

A very important aspect that emerges from the solution in closed universe must be emphasized. As the spin is included in the angular momentum $l$, the minimum value that $\omega$ is 2 . So, photons have a zero point energy of 2, which can be understood in terms of one unit of spin energy and one of kinetic, either orbital or radial.

\section{STRESS DUE TO MAXWELLIAN FIELD}

With the solutions at hand, the components of the energymomentum tensor are easily calculated. The energymomentum tensor is of course traceless, and it is more transparent to write in terms of the energy density and pressure, and after an integration over the solid angle the

Author for Correspondence: U. Khanal, Central Department of Physics, Tribhuvan University, Kirtipur, Nepal. 
relations are:

$$
\begin{aligned}
& 4 \pi \rho a^{4} \sin ^{2} z=\left|\frac{d R}{d z}\right|^{2}+\omega^{2}|R|^{2}+\frac{l(l+1)}{\sin ^{2} z}|R|^{2}, \\
& 4 \pi P_{z} a^{4} \sin ^{2} z=\left|\frac{d R}{d z}\right|^{2}+\omega^{2}|R|^{2}-\frac{l(l+1)}{\sin ^{2} z}|R|^{2}, \\
& 4 \pi\left(P_{\theta}+P_{\phi}\right) a^{4} \sin ^{2} z=\frac{2 l(l+1)}{\sin ^{2} z}|R|^{2} .
\end{aligned}
$$

One can use the equation of motion to write

$\frac{d^{2}|R|^{2}}{d z^{2}}-2\left|\frac{d R}{d z}\right|^{2}+2 \omega^{2}|R|^{2}=\frac{2 l(l+1)}{\sin ^{2} z}|R|^{2}$, whence the energy density can be rewritten as

$$
4 \pi \rho a^{4} \sin ^{2} z=\frac{d^{2}|R|^{2}}{2 d z^{2}}+2 \omega^{2}|R|^{2}
$$

to determine the normalization for R. Integrating Eq. (2.3) over $\mathrm{z}$ from 0 to $\pi$, we get

$E a^{4}=2 \omega^{2}$

where R has been normalized to unity and and the factor 2 accounts for the two helicity states of photons. As the volume of the closed universe is $V=\int_{0}^{\pi} \sin ^{2} z d z=2 \pi^{2}$, we can write the average co-moving energy density as $\rho_{\omega, l} a^{4}=\frac{\omega^{2}}{\pi^{2}}$. Summing this over $l$ and $\omega=n+l+1$, after including the Planck function, gives the average energy density as

$\rho a^{4}=\frac{1}{\pi^{2}} \sum_{n=0}^{\infty} \frac{(n+1)(n+2)^{2}}{e^{n+2}-1}=0.422336$.

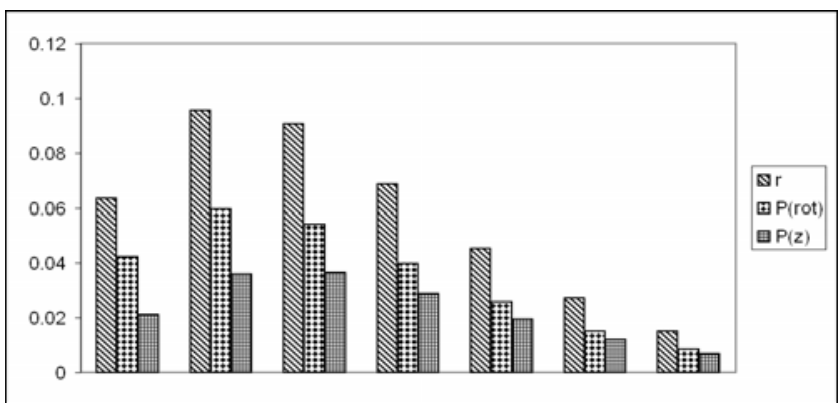

Fig. 1: The spectrum of the total energy density $\rho$ (black bar), the total rotational internal energy density $P_{\theta}+P_{0}$ (white bar) and the translational internal energy density $P_{z}$ of ${ }^{\theta}$ photons in a closed universe as a function of the energy quantum number $n$. In the large $n$ limit this distribution reverts to Planckian, but at low values of $n$ there is strong deviation, particularly a finite energy for $n=0$.

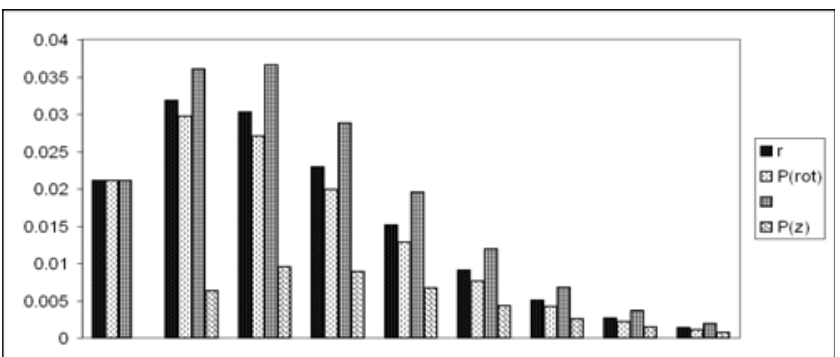

Fig. 2: The anistropy of Maxwellian stress in closed universe. Plot of $\mathrm{r} / 3$ (dark gray bar), $\left(P_{\theta}+P_{\phi}\right) / 2$ (white bar), $P_{z}$ (black bar) and $P_{z}-\left(P_{\theta}+P_{\phi}\right.$ )/2 (light gray). If the stress were equal, the first three quantities should have been the same. The radial pressure is more than the tangential one, and the last quantity plotted is the difference between these two. Only the $n=0$ mode is isotropic.
For continuous $n$, when the summation is replaced by integral, then in the limit $n \rightarrow \infty$, we recover the Planckian spectrum from the result Eq. (2.5). At low frequencies however, there is a strong deviation from Planckian, as there is a lower cut-off at $\omega=2$.

The tangential pressures can be interpreted as the energy locked as rotational internal energy; viz., the average rotational energy is

The tangential pressure that remains is $P_{z} a^{4}=\left(\rho-P_{\theta}-P_{\phi}\right) a^{4}=0.168334$. The spectral distribution of these energies resulting from Eqs. (2.4) to (2.6) are shown in Fig. 1. The results give rise to anisotropic pressure due to free photons in the closed universe. Fig. 2 shows the anisotropy. It is seen that the $n=0$, or $\omega=2$ mode is the only one that produces isotropic stress.

\section{CONCLUSIONS}

We have discussed the stress tensor of the free Maxwell field in the Friedmann-Robertson-Walker universe. The same analytic solutions can be used to represent the three cases of closed, flat and open universes, by analytic continuation throughout the complex z-plane and taking the appropriate limits. The closed universe can be represented on the real axis, the open on the imaginary and flat in the overlap region near $z \rightarrow 0$. It is also seen that most of the photon energy density of the closed universe is tied up in the tangential pressure as rotational energy, giving rise to anisotropy. As any gravitationally bound cosmological structures like the galaxy can be considered as a patch of closed universe embedded in a uniform background, it lends credence to the contention that such structures are fundamentally supported by rotation. So it appears that the intrinsic spin angular momentum of quantum fields are the origin of the angular momenta of galaxies. These preliminary results will have to be confirmed by deeper analysis and simulation. These aspects are being investigated, and will be reported in future work.

\section{REFERENCES}

1. Newman, E.T. and Penrose. 1962. R, J. Math. Phys. 3: 56.

2. Chandrasekhar, S. 1975. Proc. R. Soc. A. 343: 289.

3. Lohia, D. and Panchapakesan, N. 1979. J. Phys. A: Math. Gen. 12: 533 .

4. Khanal, U. 1985. Phys. Rev. D. 32: 879.

5. Chandrasekhar, S. 1983. Mathematical Theory of Black Holes. Oxford, Claredon Press.

6. Khanal, U. 2006. Class. Quantum. Grav. 23: 4353.

7. Khanal, U. 2006. "Comments on the Dirac Field in FriedmannRobertson-Walker Spacetime”, The Abdus Salam International Centre for Theoretical Physics, preprint no: IC/2006/136.

8. Abramowitz, M. and Stegun, I.A. 1976. Handbook of Mathematical Functions. New York, Dover. 